\title{
Hip arthroscopy has good clinical outcomes in the treatment of osteoid osteoma of the acetabulum
}

\author{
Guanying Gao, Ruiqi Wu, Rongge Liu, Yingfang Ao, Jianquan Wang ${ }^{*}$ and Yan Xu*
}

\begin{abstract}
Background: Osteoid osteoma $(\mathrm{OO})$ of the acetabulum is a relatively rare disease. However, the the clinical outcomes of hip arthroscopy for treatment of $\mathrm{OO}$ of the acetabulum are still uncertain.

Methods: We evaluated consecutive patients who were diagnosed with $\bigcirc \bigcirc$ of the acetabulum and who underwent hip arthroscopy at our hospital between January 2013 and March 2020. All patients underwent a preoperative physical examination. Preoperative supine anteroposterior hip radiography, cross-table lateral radiographs, computed tomography $(\mathrm{CT})$, and magnetic resonance imaging were performed in all patients. The alpha angle and lateral center-edge angle were measured before surgery. Supine anteroposterior hip radiography and $C T$ were performed in all patients postoperatively. Preoperative patient-reported outcomes (PROs), including Visual Analog Scale (VAS), the International Hip Outcome Tool-12 (iHOT-12) and modified Harris Hip Score (mHHS), and PROs at final follow-up were evaluated.
\end{abstract}

Results: A total of 6 patients (mean age, 18.7 years; age range, 6-31 years; 5 males and 1 females) were included in this study. The average follow-up period after surgery was 28.3 months (range, 6-90 months). Before surgery, the mean $\mathrm{mHHS}$ was $45.2 \pm 10.5$ (range, 33-56), the mean iHOT-12 was $33.3 \pm 14.5$ (range, 13-49), and mean VAS was $8.2 \pm 1.0$ (range, 7-9). At one month after surgery, mean mHHS was 78.7 \pm 1.9 (range, 77-81), iHOT-12 was $71.0 \pm 4.5$ (range, 68-80), and mean VAS was 0. At the final post-operative follow-up, mean mHHS was $89.2 \pm 2.1$ (range, 8691), iHOT-12 was $93.5 \pm 5.0$ (range, 88-98), and mean VAS was 0. All results, except VAS between one month after surgery and at final follow-up, demonstrated statistically significant improvement $(P<0.05)$. One patient underwent revision surgery.

Conclusions: Hip arthroscopy has good clinical outcomes in the treatment of $\mathrm{OO}$ of the acetabulum. Further study on the mechanism of secondary femoroacetabular impingement (FAI) caused by $\bigcirc \bigcirc$ of the acetabulum is needed. More cases of arthroscopic excision and longer follow-up are also needed to better prove the clinical outcomes of hip arthroscopy for $\mathrm{OO}$ of the acetabulum.

Keywords: Osteoid osteoma, Hip, Arthroscopy, Patient-reported outcomes

\footnotetext{
*Correspondence: kenyl417@vip.sina.com; yanxu@139.com

Institute of Sports Medicine, Beijing Key Laboratory of Sports Injuries, Peking University Third Hospital, 49 North Garden Road, Haidian District, 100191 Beijing, China
}

C C The Author(s). 2021 Open Access This article is licensed under a Creative Commons Attribution 4.0 International License, which permits use, sharing, adaptation, distribution and reproduction in any medium or format, as long as you give appropriate credit to the original author(s) and the source, provide a link to the Creative Commons licence, and indicate if changes were made. The images or other third party material in this article are included in the article's Creative Commons licence, unless indicated otherwise in a credit line to the material. If material is not included in the article's Creative Commons licence and your intended use is not permitted by statutory regulation or exceeds the permitted use, you will need to obtain permission directly from the copyright holder. To view a copy of this licence, visit http://creativecommons.org/licenses/by/4.0/. The Creative Commons Public Domain Dedication waiver (http://creativecommons.org/publicdomain/zero/1.0/) applies to the data made available in this article, unless otherwise stated in a credit line to the data. 


\section{Background}

Osteoid osteoma (OO) is a small, benign, osseous neoplasm characterized by a nidus surrounded by reactive sclerotic bone with a size usually less than $20 \mathrm{~mm}$ [1-3]. Patients often present with local pain, worsening pain, pain at night, and pain that is relieved by non-steroidal anti-inflammatory drugs (NSAIDs) [4]. OO can be diagnosed using a combination of plain radiography, technetium-99 m bone scans, computed tomography (CT) scans, and magnetic resonance (MR) images [1]. Most cases of $\mathrm{OO}$ occur in the long bones of the lower extremities of patients in the second and third decades of life [5]. The femur and tibia are affected in $>50 \%$ of cases; however, this type of tumor is rare in the pelvis and is difficult to diagnose [6-8]. OO of the acetabulum is even more rare. In recent literature, we found several case reports on treatment for $\mathrm{OO}$ of the acetabulum [1, 5, 9-23]. Minimally invasive percutaneous techniques, including $\mathrm{CT}$-guided approaches and ablation using radiofrequency or lasers, as well as arthroscopic excision techniques for $\mathrm{OO}$ in acetabulum, have been described.

The purpose of this study was to evaluate the clinical outcomes of hip arthroscopy in the treatment of $\mathrm{OO}$ of the acetabulum. We hypothesized that hip arthroscopy could relieve symptoms, improve function and prevent recurrence in the treatment of $\mathrm{OO}$ of the acetabulum.

\section{Patients and methods}

\section{Patients}

We evaluated consecutive patients who were diagnosed with $\mathrm{OO}$ of the acetabulum and who underwent hip arthroscopy for treatment at our hospital between January 2013 and March 2020. The inclusion criteria were as follows: (1) patients who were diagnosed with $\mathrm{OO}$ of the acetabulum by clinical findings, plain radiography, CT, and MR images; and (2) underwent hip arthroscopy with (3) postoperative pathological confirmation of OO. Patients who could not complete the clinical follow-up were excluded from the study. All participants signed informed consent. The study was approved by the Ethics Committee of the Third Hospital of Peking University. All methods were performed in accordance with the guidelines and regulations of the Ethics Committee of the Third Hospital of Peking University.

\section{Physical examination and radiographic assessment}

All patients underwent a thorough and systematic physical examination, including specific tests previously described for diagnosing hip diseases [24]. Flexion, adduction, and internal rotation (FADIR) or flexion, abduction, and external rotation (FABER) tests were considered positive if hip or groin pain was elicited when the hip was placed at $90^{\circ}$ of flexion, followed by adduction and internal rotation, or flexion, abduction, and external rotation [25]. Supine anteroposterior hip radiography, cross-table lateral radiography, $\mathrm{CT}$, and $\mathrm{MR}$ images were performed on all patients preoperatively (Figs. 1 and 2). Cross-table lateral radiography and CT were performed on all patients postoperatively. The preoperative alpha angle and lateral center-edge angle (LCEA) were measured as described previously [26, 27].

\section{Surgical technique and postoperative rehabilitation protocol}

One surgeon with more than 10 years of experience performed standard hip joint arthroscopy on all patients. All surgeries were performed using a standard supine approach as described by Gao et al. [28]. The operation was performed under spinal anesthesia. In brief, a

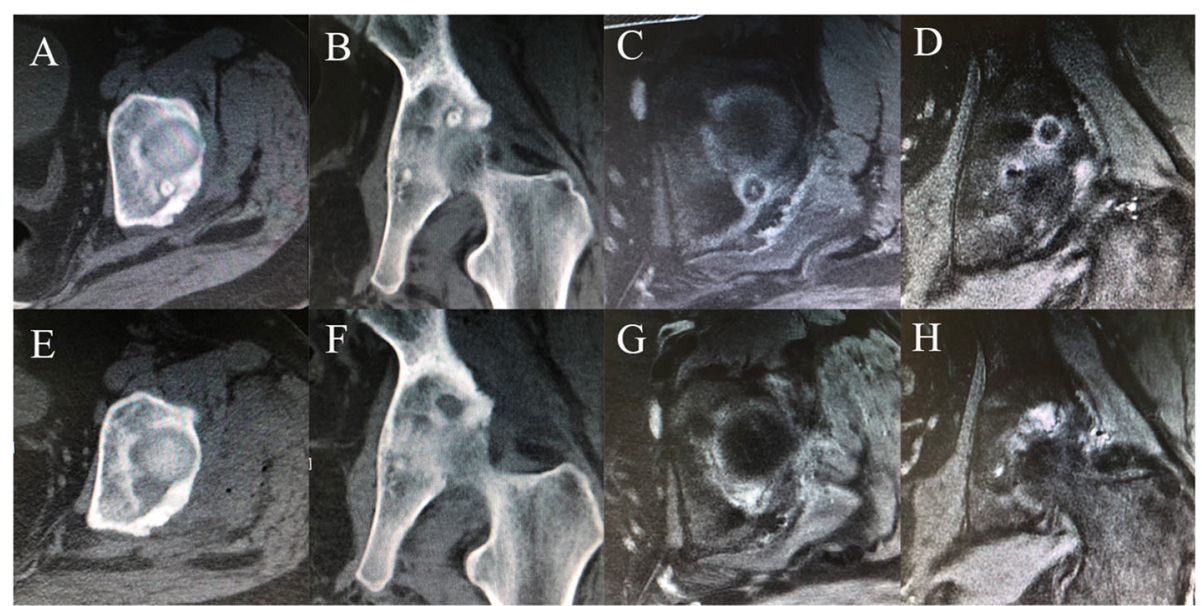

Fig. 1 Preoperative and postoperative CT and MRI of one patient diagnosed with $\mathrm{OO}$ of the acetabulum in zone 4. A-D. Preoperative axial CT, coronal CT, axial MRI and coronal MRI showed the location of OO. E-H. Postoperative axial CT, coronal CT, axial MRI and coronal MRI showed the excision of $\mathrm{OO}$ 


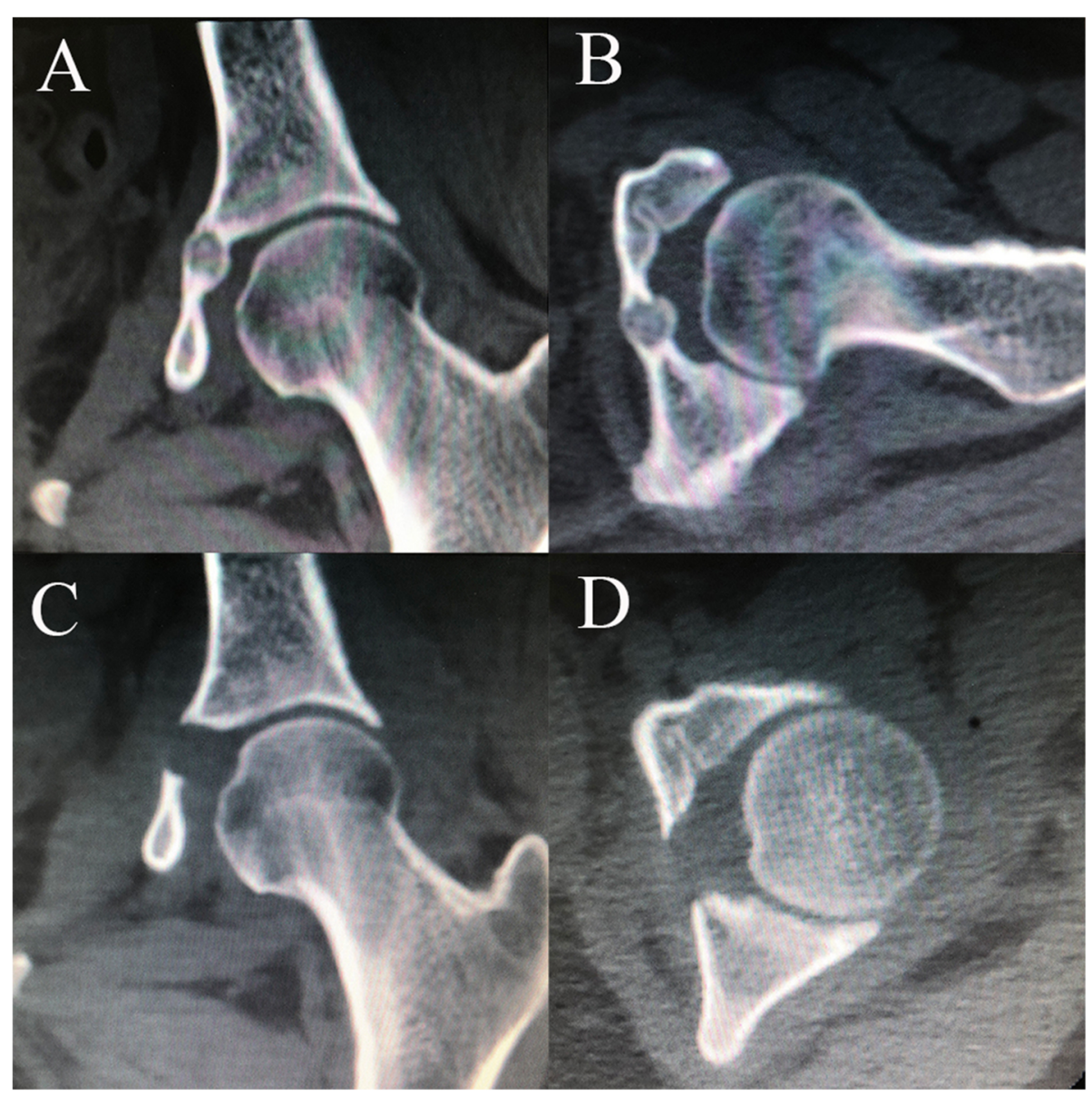

Fig. 2 Preoperative and postoperative $C T$ of one patient diagnosed with $\bigcirc \bigcirc$ of the acetabulum in zone 6 . A, C. Preoperative and postoperative coronal CT. B, D. Preoperative and postoperative axial CT

detailed inspection of the central compartment was performed to assess the acetabular rim, acetabular labrum, articular cartilage, and ligamentum teres. Labral repair or labral debridement was performed according to the nature of injury. If a cam bump in the head-neck junction or acetabular overcoverage was identified, femoral osteoplasty or acetabuloplasty was performed. The degree of cartilage damages was assessed according to the Outerbridge classification system [29]. To identify the location of $\mathrm{OO}$, we increased the force of traction for better exposure and vision. After $\mathrm{OO}$ was identified, a fine guide wire was placed to mark the nidus. Then, the $\mathrm{C}$-arm was used to confirm that the identified nidus matched what was seen on preoperative radiographic images. The tumor and surrounding sclerotic bone tissue were then removed using a narrow bone knife and an abrasive drill until normal cancellous bone was reached. Radiofrequency was used to stabilize the cartilage around the tumor. Cartilage deficiency following excision was not addressed because the resected area was relatively small. The posterior area of the hip is usually difficult to access in arthroscopy. A 70 degrees arthroscope and flexible instruments could help remove lesions. The location of $\mathrm{OO}$ was recorded according to a geographic zone method described by Ilizaliturri et al. [30]. The acetabulum was divided into six zones as follows: the anterior inferior (zone 1), the anterior superior (zone 2), the middle superior (zone 3 ), the posterior superior (zone 4), the posterior inferior (zone 5) and the middle inferior (zone 6; cotyloid fossa). After the treatment of the central compartment, the lower extremities were relaxed, and the arthroscope was inserted into the peripheral compartment. Capsular closure was routinely performed at the end of surgery.

A routine postoperative rehabilitation protocol with modifications was used, as described by Gao et al. [28] In brief, ankle pump, quadriceps strengthening and other isometric exercises were initiated 1 days after surgery. Hip passive range of motion (ROM) exercises were started as tolerated three days after surgery. Partial weight bearing with crutches was started 3 days after surgery, and passive ROM exercises and active ROM 
exercises were performed as tolerated after 2 postoperative weeks. Patients were encouraged to advance to full weight bearing by 4 postoperative weeks, and we aimed to restore symmetrical hip ROM 4 weeks after surgery. Patients were permitted to begin jogging and advance to running 3 months after surgery.

\section{Clinical evaluation}

Preoperative patient-reported outcomes (PROs) and PROs one month after surgery and at final follow-up were obtained, including visual analog scale (VAS) for pain, the International Hip Outcome Tool-12 (iHOT-12) and modified Harris Hip Score (mHHS). We used thresholds defined for PROs commonly used in the hip preservation literature. For the mHHS, the minimal clinically important difference (MCID) was defined as 8 by Kemp et al. [31], and the patient acceptable symptom state (PASS) score was defined as 74 by Chahal et al. [32]. For iHOT-12, the MCID was determined as 13 by Martin et al. [33] and the PASS was determined to be 63 by Nwachukwu et al. [34]. Complications and revision hip arthroscopy were recorded.

\section{Statistics}

A two-tailed paired t-test was used to evaluate significance between preoperative and postoperative PROs. $P$ values of $<0.05$ were considered statistically significant. All statistical analyses were performed with SPSS Statistics, version 22 (IBM).

\section{Results}

As shown in Table 1, a total of 6 patients (mean age, 18.7 years; age range, $6-31$ years; 5 males and 1 females)

Table 1 Demography of patients $(n=6)$

\begin{tabular}{ll}
\hline Parameter & Data \\
\hline Age, y, mean (range) & $18.7(6-31)$ \\
Mex & \\
Female & $5(83.3 \%)$ \\
BMl, kg/m ${ }^{2}$, mean (range) & $1(16.7 \%)$ \\
FADIR test & $20.6(12.4-33.2)$ \\
Positive & \\
Negative & $4(66.7 \%)$ \\
FABER test & $2(33.3 \%)$ \\
Positive & \\
Negative & $5(83.3 \%)$ \\
Duration of pain (range) & $1(16.7 \%)$ \\
Alpha angle (range) & $17.5(6-36)$ \\
LCEA (range) & $62.4(50.6-79.6)$ \\
\hline $\begin{array}{l}\text { NOTE. Unless otherwise specified, data are numbers of patients, with } \\
\text { percentages in parentheses }\end{array}$ & $33.4(28.1-40.6)$ \\
\hline
\end{tabular}

were included in this study. There were five cases of left-sided $\mathrm{OO}$ and one case of right-side $\mathrm{OO}$. The mean body mass index (BMI) was 20.6 (range, 12.4-33.2). The mean duration of pain before surgery in our hospital was 17.5 months (range, 6-36 months). Five patients $(83.3 \%)$ experienced worsening of pain at night, while one patient experienced the same degree of pain during the day compared with at night. Five patients $(83.3 \%)$ achieved pain relief after taking NSAIDs, while one patient did not take NSAIDs. Three patients (50\%) underwent previous surgery at another hospital and underwent revision surgery at our hospital. OO of the acetabulum in 2 of these 3 patients was misdiagnosed, and these 2 patients only underwent femoral osteoplasty and labral repair as their primary surgery. One patient had a correct diagnose of $\mathrm{OO}$ of the acetabulum and underwent radiofrequency ablation guided by $\mathrm{CT}$ at another hospital. However, none of these three patients achieved pain relief after primary surgery; thus, they attended our hospital. The FADIR test, as evaluated by the treating physician, was positive in 4 patients $(66.7 \%)$, while the FABER test was positive in 5 patients $(83.3 \%)$. In addition, 3 patients experienced tenderness in the groin area, 2 patients experienced tenderness in the posterior hip, 2 patients experienced tenderness over the greater trochanter, 1 experienced tenderness in the sacroiliac joint and 1 experienced tenderness in the posterior superior iliac spine. Mean preoperative alpha angle and LCEA were $62.4 \pm 12.9^{\circ}$ (range, 50.6-79.6) and $33.4 \pm 4.5^{\circ}$ (range, 28.1-40.6), respectively.

The arthroscopic and radiographic diagnoses of patients were shown in Table 2. Among these 6 patients, 4 patients $(66.7 \%)$ were diagnosed with combined femoroacetabular impingement (FAI), one patient was diagnosed with Tonnis grade 1 osteoarthritis (OA) by anteroposterior hip radiography, and two patients were found to have a periosteal reaction in the joint surface of the acetabulum (Fig. 3). All 6 patients underwent arthroscopic excision of OO, 4 patients underwent femoral osteoplasty, 1 patients underwent acetabuloplasty, and 2 patients underwent labral repair. $\mathrm{OO}$ of the acetabulum was located in zone 5 in 4 patients $(66.7 \%)$, in zone 4 in 1 patient (16.7\%), and in zone 6 in 1 patient (16.7\%). Two patients (33.3\%) had Outerbridge I or II femoral cartilage damages, 1 (16.7\%) who had Outerbridge IV femoral cartilage damages, 1 (16.7\%) who had Outerbridge II acetabular cartilage damages, and 3 patients (50\%) who had Outerbridge III acetabular cartilage damages.

The average follow-up period after surgery was 28.3 months (range, 6-90 months). Pain disappeared immediately after surgery in all patients. As shown in Table 3, before surgery, the mean mHHS was $45.2 \pm 10.5$ (range, 33-56), the mean iHOT-12 was $33.3 \pm 14.5$ (range, 13- 
Table 2 Diagnosis and arthroscopic findings

\begin{tabular}{ll}
\hline & Data \\
\hline Diagnosis & \\
CO of the acetabulum & $6(100 \%)$ \\
Pincer impingement & $4(66.7 \%)$ \\
Acetabular labral tear & $1(16.7 \%)$ \\
Osteoarthritis & $3(50 \%)$ \\
Location of OO & $1(16.7 \%)$ \\
Zone 4 & \\
Zone 5 & $1(16.7 \%)$ \\
Zone 6 & $4(66.7 \%)$ \\
Degree of femoral cartilage damages & $1(16.7 \%)$ \\
0 & \\
I & $3(50 \%)$ \\
II & $1(16.7 \%)$ \\
III & $1(16.7 \%)$ \\
IV & 0 \\
Degree of acetabular cartilage damages & $1(16.7 \%)$ \\
0 & \\
I & $2(33.3 \%)$ \\
II & 0 \\
\hline IV & $1(16.7 \%)$ \\
\hline
\end{tabular}

NOTE. Unless otherwise specified, data are presented as numbers of patients, with percentages in parentheses

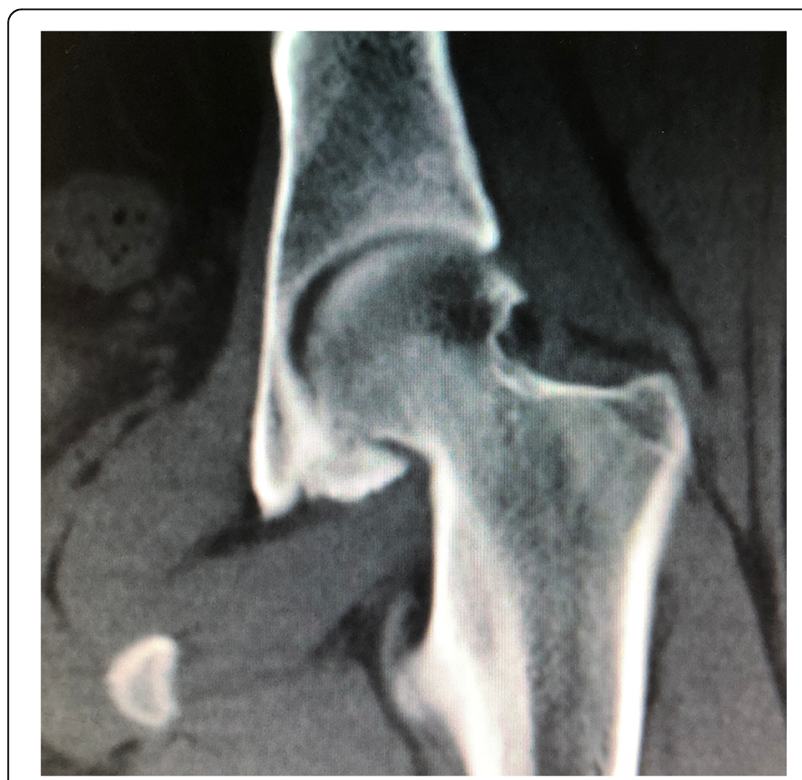

Fig. 3 Coronal $\mathrm{CT}$ showing $\mathrm{OO}$ and a periosteal reaction in the joint surface of the acetabulum
Table 3 Patient-reported outcomes

\begin{tabular}{llll}
\hline PROs & Before surgery & $\begin{array}{l}\text { One month } \\
\text { after surgery }\end{array}$ & Final follow-up \\
\hline mHHS & $45.2 \pm 10.5$ & $78.7 \pm 1.9$ & $89.2 \pm 2.1$ \\
iHOT-12 & $33.3 \pm 14.5$ & $71.0 \pm 4.5$ & $93.5 \pm 5.0$ \\
VAS & $8.2 \pm 1.0$ & 0 & 0 \\
\hline
\end{tabular}

NOTE. Values are presented as mean \pm standard deviation

49), and mean VAS was $8.2 \pm 1.0$ (range, 7-9). At one month after surgery, the mean mHHS was $78.7 \pm 1.9$ (range, 77-81), the mean iHOT-12 was $71.0 \pm 4.5$ (range, 68-80), and mean VAS was 0. At the final postoperative follow-up, the mean mHHS was $89.2 \pm 2.1$ (range, 86-91), the mean iHOT-12 was $93.5 \pm 5.0$ (range, 88-98), and mean VAS was 0. All results, except VAS between one month after surgery and at final follow-up, demonstrated statistically significant improvement $(P<$ 0.05). All patients surpassed the 2 and achieved the PASS for mHHS and iHOT-12 one month after surgery and at final follow-up. No complications were recorded during the study period. One patient still had pain after surgery and underwent revision arthroscopy because of excision at the wrong position. This patient achieved a complete pain relief after revision surgery.

\section{Discussion}

In this study, we found that hip arthroscopy has good clinical outcomes in the treatment of $\mathrm{OO}$ of the acetabulum. Pain disappeared immediately after surgery in all patients. The mHHS and iHOT-12 improved significantly one month after surgery and at final follow-up. All patients surpassed the MCID and achieved the PASS for mHHS and iHOT-12 one month after surgery and at final follow-up. VAS improved significantly one month after surgery. There was no significant difference in VAS one month after surgery compared with at final followup. The pain disappeared immediately after surgery in all patients.

$\mathrm{OO}$ of the acetabulum can be difficult to diagnose. A delay in diagnosis may lead to muscle atrophy, tenderness, localized swelling, possible contractures, articular damage and early OA [6]. Previous studies have proven the effectiveness of NSAIDs for the treatment of OO, and cases of spontaneous healing of $\mathrm{OO}$ treated with NSAIDs have been reported [4, 35]. In this study, patients with $\mathrm{OO}$ of the acetabulum can also achieved pain relief using of NSAIDs. In recent researches, percutaneous resection guided by CT scan, radiofrequency ablation, arthroscopy-assisted radiofrequency ablation, and arthroscopic excision for the treatment of $\mathrm{OO}$ of the acetabulum have been reported $[1,5,9-13,15,17-19$, 22]. With CT-guided ablation, destruction of the articular cartilage around the lesion is unavoidable, and a specimen for pathologic examination may not be obtainable 
because of thermal damage $[8,19,36]$. Mortensen et al. reported a 15 -year-old male who was treated with radiofrequency ablation for $\mathrm{OO}$ of the acetabulum after failure of primary hip arthroscopy for misdiagnosed FAI. The patient reported high satisfaction and minimal pain at 3 years of follow-up, which proved the feasibility of CT-guided percutaneous radiofrequency ablation. The advantages of arthroscopy are less surgical damage, accurate targeting and excision of the lesion, and treatment of any resultant cartilage damage [12]. Synovectomy can be also be performed during arthroscopic lesion removal, which may prevent cartilage damage, speed up healing, and immediately relieve pain [4]. Dai et al. [20] retrospectively evaluated 25 patients who underwent arthroscopic excision for hip OO, including 22 cases on femoral side and 3 in the acetabulum. They reported great improvement in mHHS and iHOT-12. However, the authors did not analyze OO of the acetabulum separately and the clinical outcomes of arthroscopic excision of $\mathrm{OO}$ of the acetabulum were thus still unclear. Eberhardt et al. [37] also reported 3 cases of $\mathrm{OO}$ of the acetabulum treated by hip arthroscopy. We found descriptions of several cases using arthroscopic excision and one case using arthroscopy-assisted radiofrequency ablation for the treatment of the $\mathrm{OO}$ of the acetabulum $[1,5,9,10,12,17,20,21,23,37]$; however, data on patients treated using by hip arthroscopy alone were scare.

In the present study, $\mathrm{OO}$ of the acetabulum in 2 of 6 patients (33.3\%) was misdiagnosed at another hospital, and the 2 patients only underwent femoral osteoplasty and labral repair only as their primary surgery. OO of the acetabulum is easy to be misdiagnosed and this feature has been previously described $[6,12,38]$. Two patients in our study underwent revision surgery after CTguided radiofrequency ablation and arthroscopic excision. Sometimes, it is indeed difficult to locate the lesion under arthroscopy. In some patients, cartilage changes could be observed on the surface of the lesion, which helped to identify the lesion. However, sometimes no abnormality is observed in cartilage.

It should be noticed that $\mathrm{OO}$ of the acetabulum in 4 of 6 patients $(66.7 \%)$ was located in zone 5 in this study. The other two cases of $\mathrm{OO}$ were located in zones 4 and 6. In the existing studies on arthroscopic treatment of OO of the acetabulum described above, two cases were located in the posterior area $[9,10]$, one was located in the posteroinferior area [13], one was located at the bottom of the acetabulum [1], one was located in the superior portion of the acetabulum [12], and one (a 10year-old boy) was located under the triradiate cartilage [17]. Thus, we concluded that, of all cases of acetabular $\mathrm{OO}$, the frequency of $\mathrm{OO}$ in the posterior acetabulum is high. In our clinical work, we need to focus on this area.
Although we used a 70 degrees arthroscope and flexible instruments to perform arthroscopic excision, it is usually difficult and time-consuming to get access to the posterior area of acetabulum, especially to zone 5. Excision of $\mathrm{OO}$ of the acetabulum in the posterior area of the hip requires suitable equipment, patience, and experience.

In this study, 4 of 6 patients $(66.7 \%)$ had concomitant FAI. We thought that FAI in these patients was secondary to OO. Three patients $(50 \%)$ had concomitant labral tear caused by secondary FAI. We thought that OO causes a repeated inflammatory reaction and bone hyperplasia, which could lead to secondary FAI. Bone hyperplasia of the acetabular fossa and relative lateral movement of the femoral head may cause secondary FAI. Further study on secondary FAI is needed in the future.

In addition, one patient in this study had sclerosis of the acetabulum and slight narrowing of the joint space and was diagnosed with Tonnis grade 1 OA. Norman et al. [39] evaluated 30 patients with intraarticular OO of the hip and found that OA developed in $50 \%$ of those patients. $\mathrm{OO}$ of the hip could stimulate an early-onset OA. Repeated inflammatory reactions that damaged cartilage may lead to OA caused by $\mathrm{OO}$ of the hip. OO of the acetabulum on the joint surface may lead to more direct and severe irritation. Moreover, two patients had a periosteal reaction in the joint surface of the acetabulum, which could be a diagnostic feature of $\mathrm{OO}$ of the acetabulum.

\section{Limitations}

This study has some potential limitations that should be noted. Firstly, this study only included a small sample size due to the rarity of $\mathrm{OO}$ of the acetabulum. Secondly, two patients were only followed up for a relatively short period of time. We found that pain disappeared immediately after surgery in all patients, so we thought that the therapeutic effect of arthroscopic excision of $\mathrm{OO}$ of the acetabulum could be achieved in a short period of time.

\section{Conclusions}

Hip arthroscopy has good clinical outcomes in the treatment of $\mathrm{OO}$ of the acetabulum. Further study on the mechanism of secondary FAI caused by $\mathrm{OO}$ of the acetabulum is needed. Furthermore, more cases of arthroscopic excision and longer follow-up are also needed to better prove the clinical outcomes of hip arthroscopy for OO of the acetabulum.

\section{Acknowledgements \\ Not applicable.}

Authors' contributions

GGY: study design, data acquisition, analyses and interpretation of data, draft of manuscripts, tables and figures. WRQ; study design, data acquisition,

analyses and interpretation of data. LRG; analyses and interpretation of data, 
manuscript with tables and figures. WJQ; study design, data acquisition, analyses and interpretation of data, manuscript with tables and figures. AYF draft of manuscripts, tables and figures. XY; study design, data acquisition, analyses and interpretation of data, manuscript with tables and figures. All authors critically reviewed and approved the final revised manuscript.

\section{Funding}

No external funds were received in support of this work.

\section{Availability of data and materials}

All relevant data supporting the conclusions are included within the article and tables. The datasets used and/or analysed during the current study available from the corresponding author on reasonable request.

\section{Declarations}

\section{Ethics approval and consent to participate}

Ethical approval The Ethics Committee of the Third Hospital of Peking University approved this study (ID number 201931802). Informed consent Informed consent was obtained from all individual participants included in the study.

\section{Consent for publication}

Not applicable.

\section{Competing interests}

The authors declare that they have no competing interests.

Received: 6 January 2021 Accepted: 17 May 2021

Published online: 28 May 2021

\section{References}

1. Ricci D, Grappiolo G, Franco M, Della Rocca F. Case report: osteoid osteoma of the acetabulum treated with arthroscopy-assisted radiofrequency ablation. Clin Orthop Relat Res. 2013:471(5):1727-32.

2. Hoffmann RT, Jakobs TF, Kubisch CH, Trumm CG, Weber C, Duerr HR, Helmberger TK, Reiser MF. Radiofrequency ablation in the treatment of osteoid osteoma-5-year experience. Eur J Radiol. 2010;73(2):374-9.

3. Saccomanni B. Erratum to: osteoid osteoma and osteoblastoma of the spine: a review of the literature. Curr Rev Musculoskelet Med. 2012;5(3):263.

4. Lee EH, Shafi M, Hui JH. Osteoid osteoma: a current review. J Pediatr Orthop. 2006;26(5):695-700.

5. Barnhard R, Raven EE. Arthroscopic removal of an osteoid osteoma of the acetabulum. Knee Surg Sports Traumatol Arthrosc. 2011;19(9):1521-3.

6. Bettelli G, Capanna R, van Horn JR, Ruggieri P, Biagini R, Campanacci M. Osteoid osteoma and osteoblastoma of the pelvis. Clin Orthop Relat Res. 1989;247(10):261-71.

7. Vanderschueren GM, Taminiau AH, Obermann WR, Bloem JL. Osteoid osteoma: clinical results with thermocoagulation. Radiology. 2002; 224(1):82-6.

8. Parlier-Cuau C, Nizard R, Champsaur P, Hamze B, Quillard A, Laredo JD. Osteoid osteoma of the acetabulum. Three cases treated by percutaneous resection. Clin Orthop Relat Res. 1999;365(8):167-74.

9. Alvarez MS, Moneo PR, Palacios JA. Arthroscopic extirpation of an osteoid osteoma of the acetabulum. Arthroscopy. 2001;17(7):768-71.

10. Khapchik V, O'Donnell RJ, Glick JM. Arthroscopically assisted excision of osteoid osteoma involving the hip. Arthroscopy. 2001;17(1):56-61.

11. Akhlaghpoor S, Aziz Ahari A, Arjmand Shabestari A, Alinaghizadeh MR. Radiofrequency ablation of osteoid osteoma in atypical locations: a case series. Clin Orthop Relat Res. 2010;468(7):1963-70.

12. Nehme AH, Bou Ghannam AG, Imad JP, Jabbour FC, Moucharafieh R, Wehbe J. Arthroscopic excision of intra-articular hip osteoid osteoma: a report of 2 cases. Case Rep Orthop. 2012;2012:820501.

13. Chang BK, Ha YC, Lee YK, Hwang DS, Koo KH. Arthroscopic excision of osteoid osteoma in the posteroinferior portion of the acetabulum. Knee Surg Sports Traumatol Arthrosc. 2010;18(12):1685-7.

14. Bosschaert PP, Deprez FC. Acetabular osteoid osteoma treated by percutaneous radiofrequency ablation: delayed articular cartilage damage. JBR-BTR. 2010;93(4):204-6.
15. Mounach A, Nouijai A, Ghozlani I, Ghazi M, Achemlal L, Bezza A, El Maghraoui A. Osteoid osteoma of the acetabulum: a case report. Rheumatol Int. 2008;28(6):601-3.

16. Tsurumoto $\mathrm{T}$, Enomoto $\mathrm{H}$, Shindo $\mathrm{H}$. Intra-articular osteoid osteoma protruding from the acetabular fossa. Mod Rheumatol. 2005;15(4):286-9.

17. Lee DH, Jeong WK, Lee SH. Arthroscopic excision of osteoid osteomas of the hip in children. J Pediatr Orthop. 2009;29(6):547-51.

18. Raux S, Kohler R, Canterino I, Chotel F, Abelin-Genevois K. Osteoid osteoma of the acetabular fossa: five cases treated with percutaneous resection. Orthop Traumatol Surg Res. 2013;99(3):341-6.

19. Mortensen AJ, Groundland JS, Tomasevich KM, Hobson TE, Randall RL, Aoki SK. Ischial osteoid osteoma: A cause of persistent hip pain in an adolescent patient with bilateral femoroacetabular impingement. Radiol Case Rep. 2021;16(5):1037-41.

20. Dai L, Zhang X, Mei Y, Gao G, Huang H, Wang C, Ju X, Xu Y, Wang J. Arthroscopic excision of intra-articular osteoid osteoma of the hip: A case series. Arthroscopy. 2021;16(4):S0749-8063(21)00326-1.

21. Marwan YA, Abatzoglou S, Esmaeel AA, Alqahtani SM, Alsulaimani SA, Tanzer M, Turcotte RE. Hip arthroscopy for the management of osteoid osteoma of the acetabulum: a systematic review of the literature and case report. BMC Musculoskelet Disord. 2015;16:318.

22. Spiker AM, Rotter BZ, Chang B, Mintz DN, Kelly BT. Clinical presentation of intra-articular osteoid osteoma of the hip and preliminary outcomes after arthroscopic resection: a case series. J Hip Preserv Surg. 2018;5(1):88-99.

23. Sahin $C, O C Y$, Ediz N, Altinay M, Bayrak AH. The safety and the efficacy of computed tomography guided percutaneous radiofrequency ablation of osteoid osteoma. Acta Orthop Traumatol Turc. 2019;53(5):360-5.

24. Gao G, Fu Q, Wu R, Liu R, Cui L, Xu Y. Ultrasound and ultrasound-guided hip injection have high accuracy in the diagnosis of femoroacetabular impingement with atypical symptoms. Arthroscopy. 2020;37(1):128-35.

25. Byrd JW. Evaluation of the hip: history and physical examination. N Am J Sports Phys Ther. 2007;2(4):231-40.

26. Barton C, Salineros MJ, Rakhra KS, Beaule PE. Validity of the alpha angle measurement on plain radiographs in the evaluation of cam-type femoroacetabular impingement. Clin Orthop Relat Res. 2011:469(2):464-9.

27. Anderson LA, Gililland J, Pelt C, Linford S, Stoddard GJ, Peters CL. Center edge angle measurement for hip preservation surgery: technique and caveats. Orthopedics. 2011;34(2):86.

28. Gao G, Zhang $X, X u Y$, Wang J. Clinical outcomes and causes of arthroscopic hip revision surgery. Sci Rep. 2019;9(1):1230.

29. Outerbridge RE. The etiology of chondromalacia patellae. 1961. Clin Orthop Relat Res 2001(389):5-8.

30. Ilizaliturri VM Jr., Byrd JW, Sampson TG, Guanche CA, Philippon MJ, Kelly BT, Dienst M, Mardones R, Shonnard P, Larson CM. A geographic zone method to describe intra-articular pathology in hip arthroscopy: cadaveric study and preliminary report. Arthroscopy. 2008;24(5):534-9.

31. Kemp JL, Collins NJ, Roos EM, Crossley KM. Psychometric properties of patient-reported outcome measures for hip arthroscopic surgery. Am J Sports Med. 2013;41(9):2065-73.

32. Chahal J, Van Thiel GS, Mather RC 3, Lee S, Song SH, Davis AM, Salata $\mathrm{M}$, Nho SJ. The patient acceptable symptomatic state for the modified harris hip score and hip outcome score among patients undergoing surgical treatment for femoroacetabular impingement. Am J Sports Med. 2015:43(8):1844-9.

33. Martin RL, Kivlan BR, Christoforetti JJ, Wolff AB, Nho SJ, Salvo JP Jr., Ellis TJ, Van Thiel G, Matsuda DK, Carreira DS. Minimal clinically important difference and substantial clinical benefit values for the 12-item international hip outcome tool. Arthroscopy. 2019:35(2):411-6.

34. Nwachukwu BU, Chang B, Beck EC, Neal WH, Movassaghi K, Ranawat AS, Nho SJ. How Should We Define Clinically Significant Outcome Improvement on the iHOT-12? HSS J. 2019;5(2):103-108.

35. Munk PL, Huk ME. Medical management of osteoid osteoma. Can J Surg. 2003:46(1):60-1 author reply 61.

36. Rosenthal DI, Hornicek FJ, Wolfe MW, Jennings LC, Gebhardt MC, Mankin HJ. Percutaneous radiofrequency coagulation of osteoid osteoma compared with operative treatment. J Bone Joint Surg Am. 1998;80(6):815-21.

37. Eberhardt $O$, von Kalle $T$, Matthis $R$, Doepner $R$, Wirth $T$, Fernandez F. A CTfree protocol to treat osteoid osteoma of the hip region in childhood and adolescence by percutaneous drilling and by hip arthroscopy. Hip Int. 2021; 3(3):1120700021996269. 
38. Franceschi F, Marinozzi A, Papalia R, Longo UG, Gualdi G, Denaro E. Intraand juxta-articular osteoid osteoma: a diagnostic challenge: misdiagnosis and successful treatment: a report of four cases. Arch Orthop Trauma Surg. 2006;126(10):660-7.

39. Norman A, Abdelwahab IF, Buyon J, Matzkin E. Osteoid osteoma of the hip stimulating an early onset of osteoarthritis. Radiology. 1986;158(2):417-20.

\section{Publisher's Note}

Springer Nature remains neutral with regard to jurisdictional claims in published maps and institutional affiliations.

Ready to submit your research? Choose BMC and benefit from:

- fast, convenient online submission

- thorough peer review by experienced researchers in your field

- rapid publication on acceptance

- support for research data, including large and complex data types

- gold Open Access which fosters wider collaboration and increased citations

- maximum visibility for your research: over $100 \mathrm{M}$ website views per year

At $\mathrm{BMC}$, research is always in progress.

Learn more biomedcentral.com/submissions 